de Daruvar, Stefan Dubel, Roanld Frank, Toby J. Gibson, Niall Haslam, Friedrich W. Herberg, Tara Hiltke, Jorg D. Hoheisel, Samuel Kerrien, Manfred Koegl, Zoltan Konthur, Bernhard Korn, Ulf Landegren, Silvere van der Maarel, Luisa Montecchi-Palazzi, Sandrine Palcy, Henry Rodriguez, Sonja Schweinsberg, Volker Sievert, Oda Stoevesandt, Michael J. Taussig, Mathias Uhlen, and Christer Wingren. A community standard format for the representation of protein affinity reagents. Molecular and Cellular Proteomics. August 2009.

\section{The contribution of the eBiokit to Bioinformatics Education in Southern Africa}
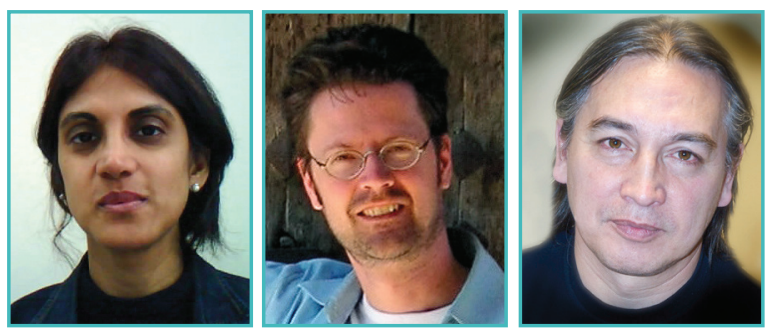

Yasmina Jaufeerally-Fakim', Hans-Henrik Fuxelius $^{2}$, Erik Bongcam ${ }^{2}$

'Faculty of Agriculture, University of Mauritius, Mauritius, ${ }^{2}$ Swedish University for Agricultural Sciences, Uppsala, Sweden

As bioinformatics has been making major progress and contributing to the development in the rest of the world, it has still not yet fully integrated the tertiary education and research sector in the countries of Southern Africa. In this context SANBio (Southern African Network for Biosciences)

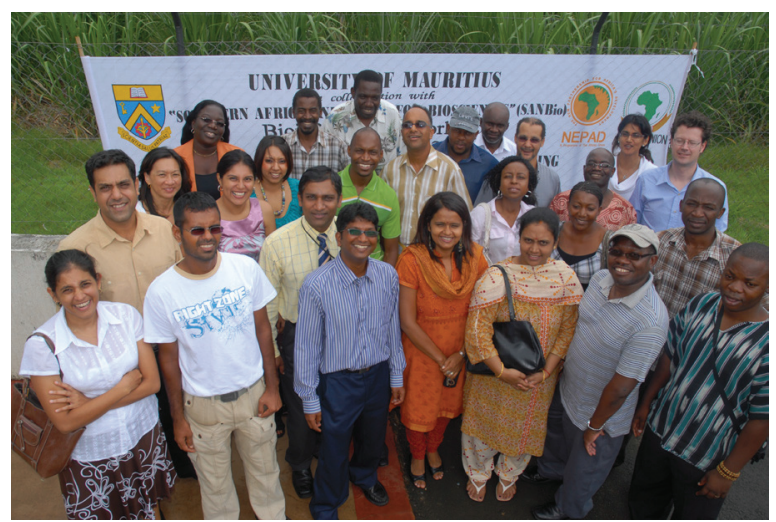

Figure 1. Students of the University of Mauritius attending the eBiokit Education.

launched a project in 2009, on capacity building in this area so as to address the immediate needs of the scientific community within the region. This project is funded under the BIOFISA program from Finland. The main challenge is to bring to the scientists the knowledge and skills required to fully tap the resources available in the public domain. With an already established network of researchers, a series of workshops were initiated so as to train those in specific fields to become familiar with some of the commonly 
used tools for DNA/protein analyses. Participants are from Malawi, Zambia, Namibia, Botswana, South Africa, Zimbabwe as well as Mauritius. Not all these countries however have easy access to the Internet or the connection can be very erratic. Although the SAFE cable has been in use for sometime, the SEACOM is relatively recent and many parts of Southern Africa do not yet enjoy good connection.

In order to address this shortcoming, the first action of the SANBio project was to put in place a training on the utilisation of the eBioKit. The latter allows a number of users to directly access to the inbuilt databases and work with the tools for analyses via their intranet. This makes teaching of a large class relatively easy with students being able to get their results very quickly. It has some of the most popular tools for alignment, phylogeny, as well as genome browsers etc. The databases include microbial, human and plants genomes. In itself it contributes to the practical sessions of a whole module in bioinformatics. The first workshop was held in Mauritius in February 2010 with Erik Bongcam-Rudloff and Hans-Henrik Fuxelius from the Swedish University for Agricultural Sciences (SLU), Uppsala, Sweden.

The material covered in this course was alignment and phylogeny software on real world data as well as homology searching on major protein databases locally stored on the eBiokit. EMBOSS and Phylip packages was primarily used for building the phylogenies and everything was accessed through the web interface to the eBiokit server. Once the process has been mastered on how to use these tools the participants can teach their colleagues on the same platform with the same material.

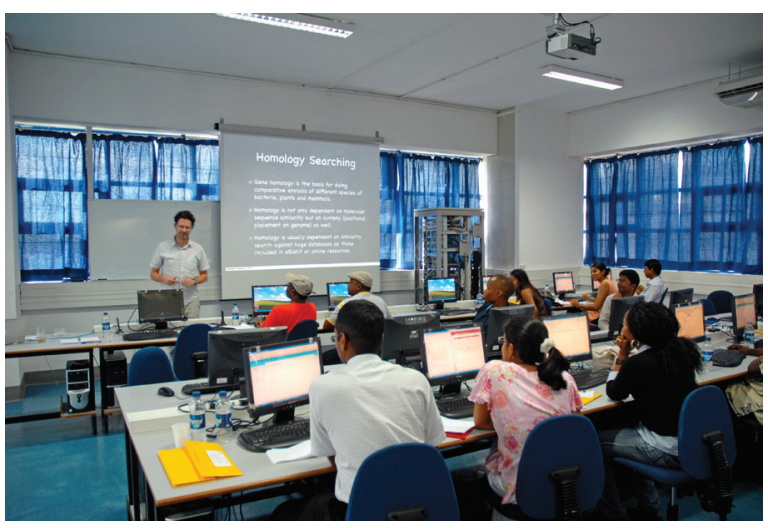

Figure 2. Dr. Hans-Henrik Fuxelius lecturing on homology searches using the eBioKit.

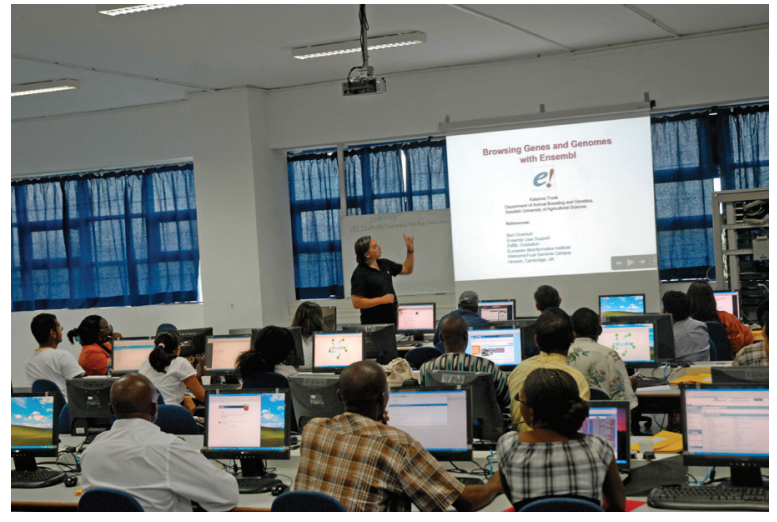

Figure 3. Dr. Erik Bongcam-Rudloff lecturing on using ensembl with the eBioKit.

Most of the participants were at their first hands-on training in using such tools and by the end they were eagerly waiting to have the kit at their own institution. Two kits have been delivered to Zimbabwe, one at the Tobacco Research Board and the other at the University of Zimbabwe. There are now two eBiokits at the University of Mauritius. The rest have been shipped to the other countries. Those who got their training are preparing to run their own local workshop; Mauritius and Malawi will hold one each for their local participants in July.

This kit has been received with great excitement and above all it is a means to allow the rapid dissemination of knowledge in Bioinformatics. Both faculty members and students are utilising the resources. It is a catalyst to further upgrade teaching and research standards of the universities in the region. The utilisation of the eBioKit will set the pace for further development in the application of Bioinformatics among scientists in Southern Africa. It is indeed a great way of providing the means for enhancing the quality of research output and above all give students the opportunity to know better the exciting world of genomes and gene expression. 